\title{
HIV-Infected Patient Diagnosed With Osteomedullary and Hepatic Syphilis on Positron Emission Tomography: A Case Report
}

\author{
Antoine Desilets $^{\mathrm{a}, \mathrm{d}}$, Frederic Arsenault ${ }^{\mathrm{b}}$, Mikhael Laskine ${ }^{\mathrm{c}}$
}

\begin{abstract}
A human immunodeficiency virus (HIV)-positive man presented with a recent history of weight loss and B symptoms. Positron emission tomography (PET) scan revealed multiple hypercaptation foci, including diffuse osteomedullary lesions and a dominant liver nodule. Syphilis screening was positive, with a rapid plasma reagin (RPR) titer of 1:1,024. Following antibiotic therapy, repeat PET scan demonstrated complete resolution of hypermetabolic lesions, arguing toward an infectious etiology. Cases of disseminated syphilis characterized on nuclear imaging typically demonstrate radiological evidence of target tissue inflammation. PET scan could however also represent a sensitive modality for evaluation of early subclinical secondary syphilis.
\end{abstract}

Keywords: Positron emission tomography; Secondary syphilis; Liver; Bone marrow

\section{Introduction}

Cases of Treponema pallidum (T. pallidum) infection across North America and Europe have risen dramatically over the last three decades [1]. This changing epidemiology is most striking in certain high-risk subgroups, such as men who have sex with men (MSM), and may be attributable to a change in sexual behaviour at the advent of highly active antiretroviral therapy (HAART) development. Studies have estimated that HIV coinfection would be present in up to $25 \%$ of new cases of syphilis in the United States [2].

Genital chancres associated with early treponemal infec-

Manuscript submitted January 16, 2019, accepted February 27, 2019

${ }^{a}$ Centre Hospitalier de l'Universite de Montreal (CHUM), 1051 rue Sanguinet, H2X 3E4, Montreal, QC, Canada

bNuclear Medicine Department, Centre Hospitalier de l'Universite de Montreal (CHUM), 1051 rue Sanguinet, H2X 3E4, Montreal, QC, Canada 'Department of Internal Medicine, Centre Hospitalier de l'Universite de Montreal (CHUM), 1051 rue Sanguinet, H2X 3E4, Montreal, QC, Canada

${ }^{\mathrm{d}}$ Corresponding Author: Antoine Desilets, Centre Hospitalier de 1'Universite de Montreal (CHUM), 1051 rue Sanguinet, H2X 3E4, Montreal, QC, Canada. Email: antoine.desilets@umontreal.ca

doi: https://doi.org/10.14740/jocmr3756 tion likely enhance risk of human immunodeficiency virus (HIV) inoculation during sexual intercourse. A higher incidence of asymptomatic primary syphilis has however been described in the HIV-positive patients. Up to $58 \%$ of HIV-positive patients would thus be diagnosed with secondary syphilis at presentation (versus $40 \%$ in the HIV-negative population) [3]. Progression from early syphilis, as evidenced by systemic dissemination of spirochetes to target organs, could thus be accelerated and have a more aggressive clinical course in this specific population [1].

Gummatous syphilis, defined as granuloma formation secondary to a delayed hypersensitivity reaction to T. pallidum, can be identified on computed tomography (CT) imaging as hypodense lesions, with or without calcifications. Other variants of tertiary syphilis may affect sanctuary sites such as the brain (neurosyphilis) or the aorta (syphilitic aortitis) [4]. Highly vascularised organs, such as the liver and bones, could also represent high-risk sites for spirochete invasion and replication.

\section{Case Report}

A 54-year-old male known for HIV/ acquired immunodeficiency syndrome (AIDS) on HAART presented to the emergency department in the context of general deterioration, posterior rib pain, progressive weight loss for the last month and a history of transient erythematous maculopapular rash. His past medical history was significant for cerebral toxoplasmosis, acute hepatitis B, gout and secondary syphilis treated with intramuscular penicillin $\mathrm{G}$.

Physical examination revealed point tenderness at the posterior eighth rib, but no evidence of fever, skin lesions, lymphadenopathy or hepatosplenomegaly. A CD4 count performed 4 months earlier showed 580 cells $/ \mathrm{mm}^{3}$ and quantitative HIV viral load was below 40 copies. Laboratory work was significant for normocytic anemia and increased inflammatory markers, with a C-reactive protein of $191 \mathrm{mg} / \mathrm{L}$ (normal $<10$ $\mathrm{mg} / \mathrm{L}$ ). Aspartate aminotransferase (AST), alanine aminotransferase (ALT), alkaline phosphatase (ALP), bilirubin, albumin and a hepatitis panel all were within normal range.

Syphilis screening was also positive, with a rapid plasma reagin (RPR) value of 1:1,024 (from a post-treatment value of 1:4, 4 months earlier). Positive RPR was deemed to be secondary to a re-infection, in contrast to failure of the initial treatment. A 2-week course of intravenous penicillin was thus 

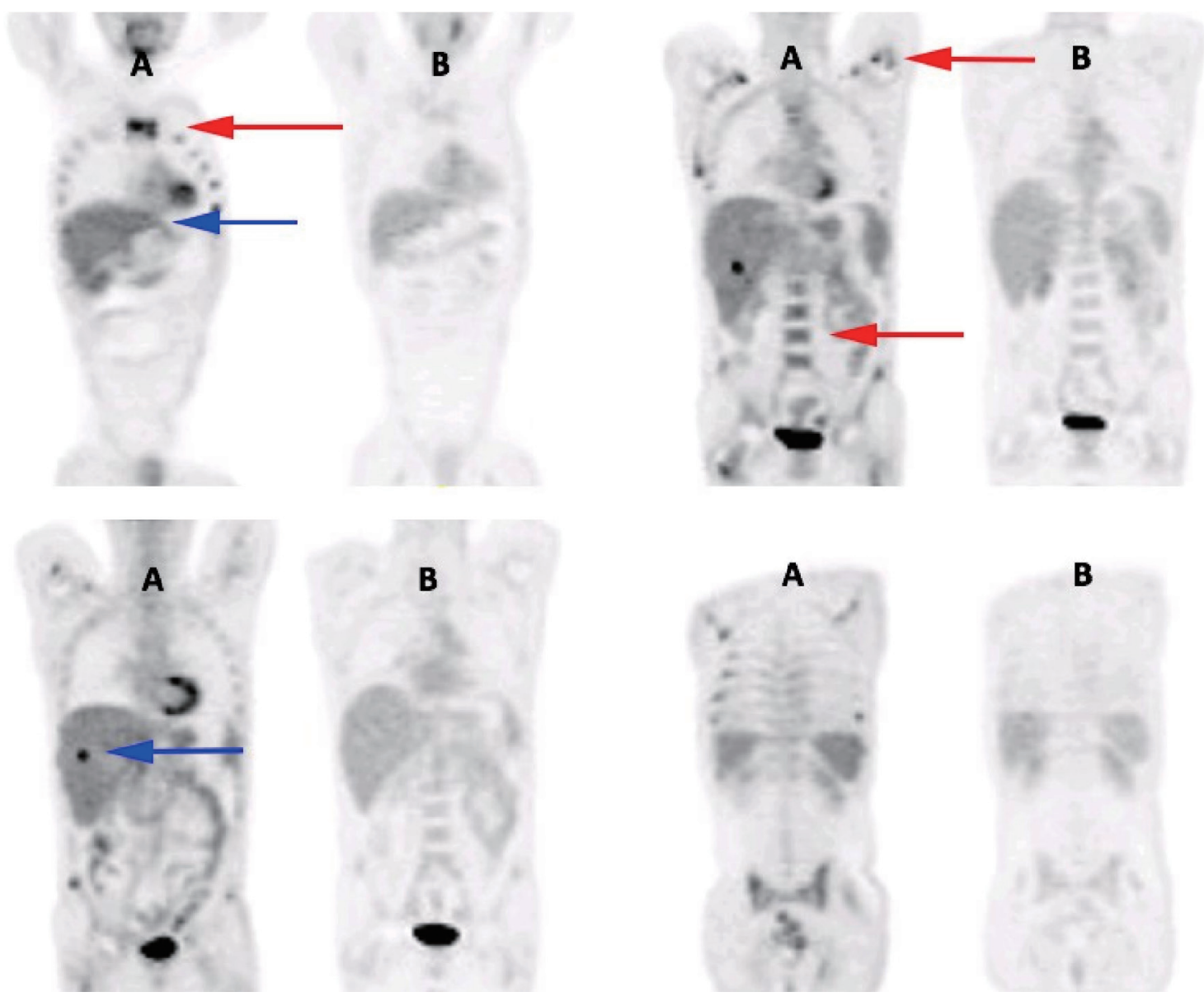

Figure 1. Initial positron emission tomography imaging (A) and results after 2 weeks of intravenous penicillin therapy (B). Red arrows indicate areas of increased osteomedullary hypermetabolism on nuclear imaging, while the blue arrows point toward a hypercaptation focus in the liver, both of which resolved following antibiotics.

initiated. The patient developed a Jarisch-Herxheimer reaction on the first day of therapy, with self-resolving fever and tachycardia.

In the context of prominent B symptoms, a positron emission tomography (PET) coupled with CT scan (PET-scan) was

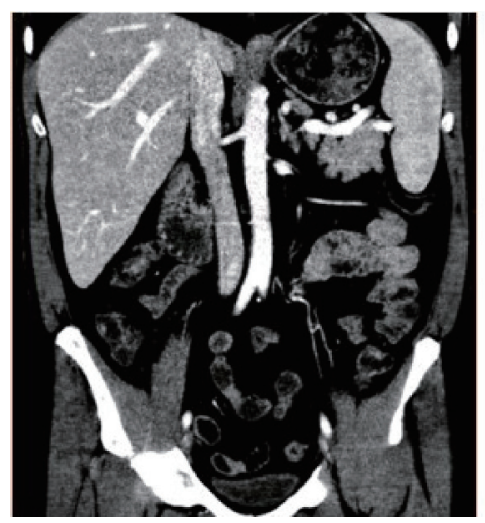

performed (Figs. 1, 2) and demonstrated multiple hypercaptation foci, including diffuse osteomedullary lesions of the axial skeleton and long bones $\left(\mathrm{SUV}_{\max } 5.2\right.$ - 8.1), perianal region hypermetabolism $\left(\mathrm{SUV}_{\max }\right.$ 7.7) as well as a dominant liver nodule $\left(\mathrm{SUV}_{\max } 8.0\right.$ - 9.7). A thoracoabdominal scan was com-

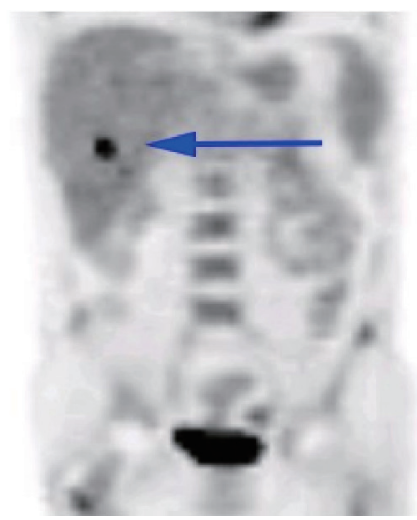

Figure 2. Radiological correlation of the segment VI liver lesion between CT abdominal scan (left) and PET (right). The blue arrow points toward a hypercaptation focus in the liver, which didn't anatomically correlate with any density changes on computed tomography. 
pleted to investigate for an underlying primary neoplasm, but failed to reveal any evidence of solid malignancy, lymphoproliferative disorder or suspicious lymphadenopathy. No radiological correlations to CT were recognised upon additional review of the PET images, except for a $17 \mathrm{~mm}$ liver hypodensity of segment VI. Rectoscopy was also negative.

Hematopoietic involvement was assessed during hospitalisation. Flow cytometry and serum protein electrophoresis were performed, with normal results; and a bone marrow biopsy was normocellular, without any evidence of lymphoproliferative disorder or myeloproliferative neoplasia. Bone marrow culture was also negative.

Considering discrepancy between radiological and nuclear imaging modalities, liver biopsy was postponed, as disseminated syphilis remained the working diagnosis despite unusual nuclear imaging results.

Follow-up PET scan 3 weeks after admission however demonstrated complete interval resolution of hypermetabolic osteomedullary and liver lesions, arguing against a metastatic neoplastic process and pointing toward an initial disseminated syphilis infection. From a clinical perspective, the patient responded well to intravenous penicillin therapy and was thus discharged from the hospital ward with follow-up appointments in microbiology.

\section{Discussion}

\section{Hepatic involvement}

Syphilitic hepatitis is a well-described pathological entity in medical literature. Classically presenting as signs of cholestasis in a patient with secondary syphilis, ALP and gammaglutamyl transferase (GGT) elevation often represent isolated findings, without signs of clinical jaundice [5]. Infection with HIV may represent a risk factor for such complication. A retrospective study of 32 cases of syphilis with HIV coinfection indeed identified laboratory evidence of hepatitis in $32 \%$ of patients [6].

Cases of inflammatory pseudohepatic tumors have also been described in association with active syphilis and low-attenuation masses on CT scan. Such lesions typically respond to systemic antibiotherapy as evidenced by interval radiological resolution. The infectious nature of the tumors can however only be assumed, as cultures of pathological samples typically fail to grow microorganisms [7].

In our present case, no liver enzyme perturbation was objectified in association with the hypermetabolic lesions on PET. Decision was made to postpone CT-guided liver biopsy while awaiting results of follow-up nuclear imaging. Liver biopsy, although not systematically indicated, may however be informative in differentiating syphilitic foci from other complications of HIV. Solid neoplasia, lymphomas, Kaposi sarcomas and mycobacterial infections thus need to be ruled out in this high-risk population.

Hypercaptation of the rectal vault was another finding evocated on PET imaging. Anal sex has previously been speculated as a mechanism of entry for syphilitic infection, from infiltration into the portal system and seeding of hepatic lobules [8].

\section{Bone involvement}

Skeletal involvement has classically been observed among cases of tertiary or congenital syphilis, such as tibia bowing associated with saber shin. Proliferative periostitis would however represent a common type of bone lesion in the context of earlier syphilis infection [9]. Although a periosteal reaction or lytic lesions can be identified through X-rays, most inflammatory foci remain radiologically silent.

Development of new radiological modalities in the last decades has allowed us to better characterize skeletal lesions in early syphilis stages. A systematic review by Park et al studied 37 patients presenting with secondary syphilis and bone involvement, diagnosed either through plain radiographs, CT scan, magnetic resonance imaging (MRI) or bone scintigraphy [10]. Only $25 \%$ of periostitis cases had abnormal findings on plain radiographs and most of the lesions (74\%) were described as multifocal. Furthermore, 11 patients had serological evidence of HIV coinfection.

While the patient in the previously reported case presented with symptomatic costal pain and diffuse skeletal hypercaptation on PET, no radiological correlation was made evident on thoracic CT scan in terms of lytic or periosteal lesions. Medullary involvement was also questioned considering the risk of HIV-related lymphoproliferative disorder, but hematological workup revealed no abnormality. Interval resolution of hypercaptation following the 2-week penicillin course however pointed toward an infectious etiology to the previously reported symptoms.

\section{Syphilis and positron emission tomography}

Other cases of disseminated syphilis characterization with PET have been described in the last decade, with disease activity involving the aorta, bones, lungs, adrenal glands or the lymphatic system [11-16]. Evaluation of the underlying etiology of PET hypercaptation can represent a diagnostic challenge for clinicians and nucleists, especially in the face of B symptoms in a HIV-infected patient.

What those cases had in common was an interval resolution of hypermetabolic lesions following prolonged penicillin therapy. Most patients however simultaneously demonstrated radiological evidence of a target tissue inflammatory response, such as lytic bone destruction or sclerotic lesions [14]. To our knowledge, no report to date has described such discordance in regards to osteomedullary hypercaptation in the absence of detectable anatomical changes by conventional radiological techniques.

In the case presented here, normal liver panel and lack of radiological or pathological correlation to osseous hypermetabolism could point toward an early systemic dissemination of T. pallidum, perhaps restricted to an initial vasculitis-associated inflammation. For obvious cost-effectiveness reasons, routine 
nuclear medicine techniques should not be employed as part of the initial evaluation of patients with secondary syphilis. Nontreponemal titers and clinical evaluation remain of paramount importance in the monitoring of treatment response. Secondary syphilis should however be included in the differential diagnosis of hypermetabolic PET lesions, including multi-organ hypercaptation foci in the absence of other imaging abnormalities.

\section{Conflict of Interest}

The authors declare that there is no conflict of interest.

\section{Informed Consent}

Patient's written informed consent has been obtained regarding the publication of this article.

\section{References}

1. Lynn WA, Lightman S. Syphilis and HIV: a dangerous combination. Lancet Infect Dis. 2004;4(7):456-466.

2. Chesson HW, Heffelfinger JD, Voigt RF, Collins D. Estimates of primary and secondary syphilis rates in persons with HIV in the United States, 2002. Sex Transm Dis. 2005;32(5):265-269.

3. CDR. Recent developments in syphilis epidemiology. CDR. 2003;13.

4. Gaslightwala I, Khara HS, Diehl DL. Syphilitic gummas mistaken for liver metastases. Clin Gastroenterol Hepatol. 2014;12(11):e109-110.

5. Manavi K, Dhasmana D, Cramb R. Prevalence of hepatitis in early syphilis among an HIV cohort. Int J STD AIDS. 2012;23(8):e4-6.

6. Crum-Cianflone N, Weekes J, Bavaro M. Syphilitic hepatitis among HIV-infected patients. Int J STD AIDS.
2009;20(4):278-284.

7. Mahto M, Mohammed F, Wilkins E, Mason J, Haboubi NY, Khan AN. Pseudohepatic tumour associated with secondary syphilis in an HIV-positive male. Int J STD AIDS. 2006;17(2):139-141.

8. Solari PR, Jones C, Wallace MR. Hepatic Lesions with Secondary Syphilis in an HIV-Infected Patient. Case Rep Med. 2014;2014:604794.

9. Reginato AJ. Syphilitic arthritis and osteitis. Rheum Dis Clin North Am. 1993;19(2):379-398.

10. Park KH, Lee MS, Hong IK, Sung JY, Choi SH, Park SO, Shin MJ, et al. Bone involvement in secondary syphilis: a case report and systematic review of the literature. Sex Transm Dis. 2014;41(9):532-537.

11. Pruzzo R, Redondo F, Amaral H, Glasinovic E, Caviedes I, Glasinovic JC. Anal and rectal syphilis on F-18 FDG PET/CT. Clin Nucl Med. 2008;33(11):809-810.

12. Kosters K, Bleeker-Rovers CP, van Crevel R, Oyen WJ, van der Ven AJ. Aortitis diagnosed by F-18-fluorodeoxyglucose positron emission tomography in a patient with syphilis and HIV coinfection. Infection. 2005;33(56):387-389.

13. Lin M, Darwish BS, Chu J. Neurosyphilitic gumma on F18-2-fluoro-2-deoxy-D-glucose (FDG) positron emission tomography: an old disease investigated with a new technology. J Clin Neurosci. 2009;16(3):410-412.

14. Wang X, Yin J, Chen X. Syphilitic bone destruction on FDG PET/CT. Clin Nucl Med. 2011;36(7):616-618.

15. Fu Z, Zhang J, Li Q, Liu M, Kang L. A case of secondary syphilis involving tonsil, pulmonary, and multiple lymph nodes: 18F-FDG PET/CT findings. Clin Nucl Med. 2015;40(4):335-337.

16. Ranganath P, Kapila R, Vadehra V, Wang Q, Capitle E, Jr., Ghesani N. Generalized lymphadenopathy and 18-fluorine fluorodeoxyglucose positron emission tomography/ computed tomography: targeting diagnostic intervention, characterizing disease extent, and assessing treatment efficacy in syphilis. Sex Transm Dis. 2015;42(2):68-70. 